\title{
Role of Crop Load in Chloroplast Ultra-structure and Zonal Chlorosis, a Physiological Disorder in 'Honeycrisp' Apple Trees
}

\author{
Teresa Eileen Snyder-Leiby ${ }^{1,2}$ and Shixiong Wang \\ SUNY New Paltz, Biology, 102 CSB, 75 Manheim Boulevard, New Paltz, \\ NY 12561
}

Additional index words. zonal chlorosis, Honeycrisp, TEM chloroplast, starch grains, SUNY New Paltz

\begin{abstract}
Honeycrisp' is a relatively new apple cultivar (Malus $\times$ domestica Borkh.) with a unique crisp fruit texture that makes it highly desirable. However, the leaves often develop a zonal chlorosis that resembles potato leafhopper damage. Other researchers have determined that the symptoms correlate with decreasing crop load rather than leafhopper damage. This study investigates the possibility that the zonal chlorosis is related to the buildup of starch grains causing rupture of chloroplasts. Transmission electron microscopy was used to document ultrastructural changes in chloroplasts in trees with three different crop loads from the beginning of the season to the end of the season. In trees with extremely low crop loads, we observed abnormalities of chloroplast membrane structure and accumulation of larger and more numerous starch grains. These appear before the appearance of chlorotic symptoms. By early August, the chloroplasts from chlorotic regions of leaves on trees with light crop loads are completely disrupted and abnormally large starch grains are found in place of chloroplasts. As the season progresses, trees with moderate and heavy crop loads showed less severe chloroplast disruption and smaller starch grain accumulation than trees with light crop load.
\end{abstract}

'Honeycrisp' is a new apple cultivar with a unique, crisp fruit texture that makes it highly desirable. As growers, Extension advisors, and researchers gain more experience with growing 'Honeycrisp', it becomes increasingly apparent that this cultivar has a number of characteristics, which present challenges for the commercial grower (Rosenberger et al., 2001, 2004). One of the challenges to growing 'Honeycrisp' is the development of zonal chlorosis that resembles potato leafhopper damage. Because misdiagnosis of the cause of these symptoms could lead to unnecessary pesticide applications, a replicated trial of commercial thinners to manipulate crop load was performed on 'Honeycrisp' trees (Schupp et al., 2001). These trees were caged to exclude insects while the study was conducted. The study

\footnotetext{
Received for publication 12 May 2006. Accepted for publication 13 Aug. 2006.

Summer Undergraduate Research Experience (SURE), SUNY New Paltz funding provided support in 2005.

We thank Jim Schupp, Biglerville Fruit Research Station, for extremely helpful discussions and tissue samples from chemically thinned trees; David Rosenberger, Superintendent of Cornell University's Hudson Valley Laboratory, for field site access and support; T. Page Owen, Connecticut College, for use of the FEI 120 TEM; Richard Christiana for laboratory assistance; and Dave Ricci and Tanya Jurcic for field assistance. ${ }^{1}$ Current address: SoftGenetics LLC, 200 Innovation Blvd., Suite 235, State College, PA 16803

${ }^{2}$ To whom reprint requests should be addressed; e-mail teresa@softgenetics.com
}

present (Das et al., 1998; Eckardt, 2003; Zeeman et al., 1998).

The objective of this study was to determine if 'Honeycrisp' trees with varying crop loads develop large starch granules in the chloroplasts, leading to chlorosis. Transmission electron microscopy (TEM) analysis was selected because it would enable visualization of potential starch accumulation and chloroplast membrane integrity. The study was initiated before publication of the study of Chen and Cheng's (2004) work that investigated the causes for zonal chlorosis.

\section{Methods}

The initial study was conducted between June and Aug. 2001 and 2003 using chemical thinners. Subsequent replications of the study with hand thinning were carried out in 2004 and 2005.

Chemical thinning to reduce crop load. 'Honeycrisp' trees on M.9 rootstocks at Cornell University's Hudson Valley Laboratory, Highland, NY, were selected. Selection was based on uniform bloom density and vegetative vigor. Leaf samples for zonal chlorosis were taken from trees in separately run studies evaluating chemical thinners (Schupp, 2003). A range of chemical thinners was applied to drip by an airblast sprayer when the largest fruits were 10 to $12 \mathrm{~mm}$ in diameter. Treatments to achieve a light, moderate, or heavy crop load on trees were applied in a randomized, complete block design with six replications. Trees with light, moderate, and heavy crop loads were selected for evaluation of chloroplast ultrastructure.

Hand thinning to reduce crop load. Trees from the Hudson Valley Laboratory were selected using the same criteria as those in the chemical thinning trials. Thinning treatments were performed 2 weeks after fruit set. Treatments to achieve a light, moderate, or heavy crop load on trees were applied in a randomized, complete block design with six replications. Light crop load was achieved by removing all but one of the apples per spur. Medium crop load was achieved by removing half of the apples per spur. No apples were removed from the heavy crop load treatment. The unthinned trees developed much less chlorosis and were used as the baseline for starch granule accumulation.
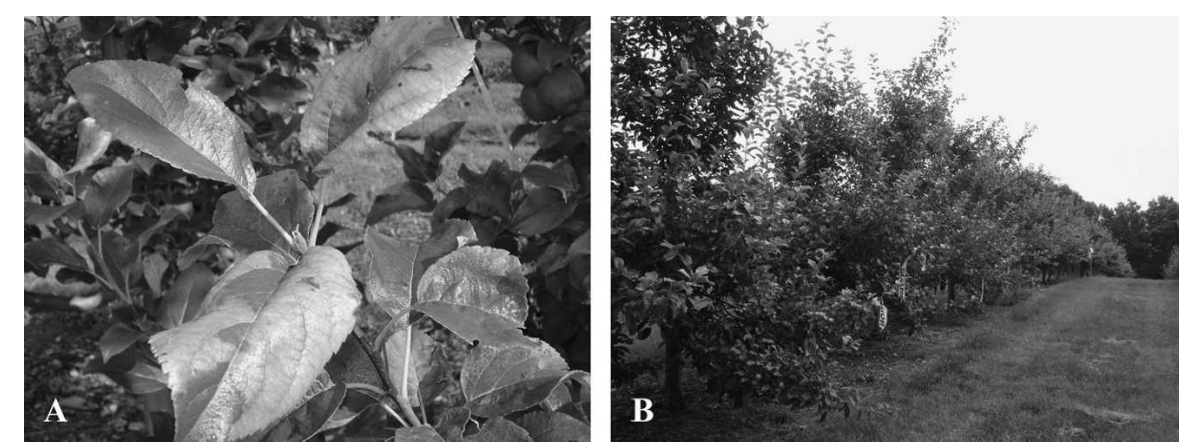

Fig. 1. (A) Zonal chlorosis symptoms in a tree with light crop load in the first week of July 2005. (B) The test sited at Cornell University's Hudson Valley Laboratory, Highland, NY. 
Electron microscopy. Figure 1A shows zonal chlorosis symptoms in a tree with light crop load in the first week of July. Circles indicate the areas of the leaf where $4.0-\mathrm{mm}$ disks were collected for TEM analysis. Figure $1 \mathrm{~B}$ is the test sited at the Hudson Valley Laboratory and illustrates the consistent size and vigor of trees in the study. A cork borer was used to take 4-mm diameter disks from Stage 3 (http://www.plantontology.org/), fully expanded leaves in the field from the areas of veinal chlorosis and chlorotic tips for TEM analysis. Analogous regions of veins and tip margins were sampled in the nonsymptomatic leaves. All samples were taken during the morning between 0800 and $1100 \mathrm{HR}$.

Tissue was immediately placed into $4 \%$ glutaraldehyde in cacodylate buffer, $\mathrm{pH} 6.8$. Tissues were processed for TEM by standard methods for plant tissues (Bozzola and Russell, 1998). Briefly, tissues were processed as follows: primary fixation in $4 \%$ glutaraldehyde/cacodylate buffer, $\mathrm{pH} 6.8$ (12 to $24 \mathrm{~h}$ at $4{ }^{\circ} \mathrm{C}$ ); three buffer rinses, $\mathrm{pH}$ 6.8; secondary fixation in $2 \%$ osmium tetroxide, $\mathrm{pH} 6.8$ ( $1 \mathrm{~h}$ at room temperature); three $\mathrm{ddH}_{2} \mathrm{O}$ rinses; $2 \%$ aqueous uranyl acetate stain en bloc ( $1 \mathrm{~h}$ at room temperature); three $\mathrm{ddH}_{2} \mathrm{O}$ rinses; dehydration series from $25 \%$ to $100 \%$ ethanol (15 to $25 \mathrm{~min}$ each); final dehydration with three exchanges of acetonitrile (20 min each at room temperature); and 2-d infiltration with decreasing volumes of acetonitrile and increasing volumes of epoxy (10.0 mL Embed-812, $8.0 \mathrm{ml}$ dodenyl succinic anhydride, $6.0 \mathrm{~mL}$ nadic methyl anhydride, $0.6 \mathrm{~mL}$ benzyl dimethyl amine; Electron Microscopy Sciences, Fort Washington, PA). Thin sections were stained with $0.1 \%$ lead citrate and $2 \%$ aqueous uranyl acetate before TEM observation. A Phillips 300 TEM (Phillips, Eindhoven, Netherlands) (2001, 2003, 2004) and an FEI 120 (FEI, Hillsboro, OR) (2005) were used for analysis and photography of palisade parenchyma and spongy mesophyll of all leaf samples. Early season samples (June) and late summer samples (mid-August) were evaluated for starch accumulation and chloroplast integrity (two samples per each crop load treatment). Over the 4 years of the study, 48 samples were evaluated using TEM.

\section{Results}

Hand thinning to reduce crop load. Results of hand thinning to reduce crop load are presented in Table 1. Hand thinning resulted in approximate loads of 100, 200, and 340 (light, moderate, and heavy, respectively) fruit per tree were comparable to crop

Table 1. Total number of apples per tree for each crop load for 2004 and $2005^{2}$.

\begin{tabular}{lcr}
\hline Crop load & \multicolumn{1}{c}{2004} & \multicolumn{1}{c}{2005} \\
\hline Heavy & $341.67 \pm 12.50$ & $316.10 \pm 16.46$ \\
Moderate & $195.00 \pm 13.23$ & $194.50 \pm 16.46$ \\
Light & $110.00 \pm 10.00$ & $91.25 \pm 22.29$ \\
\hline
\end{tabular}

${ }^{2}$ Crop load data for 2001 and 2003 were not collected but are comparable to 2004 and 2005 . loads obtained in previous years by chemical thinning. All crop loads resulted in chlorosis. The chlorosis was most severe in the trees with light crop load and least severe in the trees with heavy crop load.

Electron microscopy. Micrographs in Figures 2 and 3 are representative areas from trees with light, moderate, and heavy crop loads. The samples illustrated were harvested on 6 June 2001 and 11 June 2005, before zonal chlorosis symptom development, and on 22 Aug. 2001 and 1 Aug. 2005 when chlorotic symptoms were present in all treatments regardless of fruit thinning method. Normal starch grains are present in chloroplasts early in the season in most treatments (Fig. 2A-E). Lipid bodies appear in the chloroplasts in all treatments in the August sampling (Fig. 3C, D). During 2001, some of the samples from trees with light crop loads have damage to the
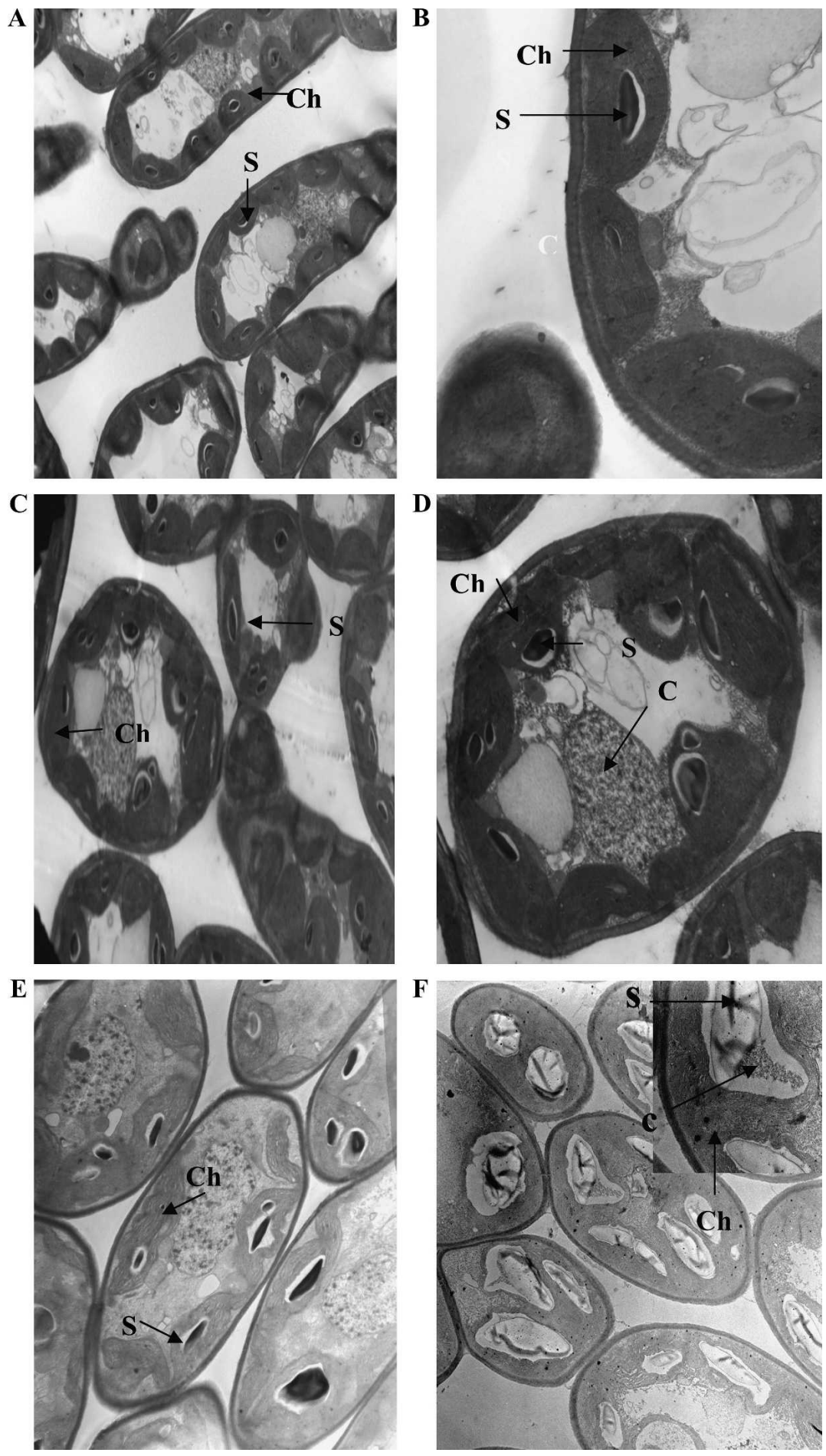

Fig. 2. Typical ultrastructure of leaves harvested in June (A, D, 2005; E, F, 2001). (A) Hand-thinned, heavy crop load, $\times 2800$; (B) hand-thinned, heavy crop load, $\times 11,000$; (C) hand-thinned, light crop load, $\times 2800$; (D) hand-thinned, light crop load, $\times 7100$; (E) chemical-thinned, heavy crop load, $\times 3900$; and (F) chemical-thinned, light crop load, $\times 3900$. Ch, chlorosplast; C, cytoplasm; S, starch grain; L, lipid. 

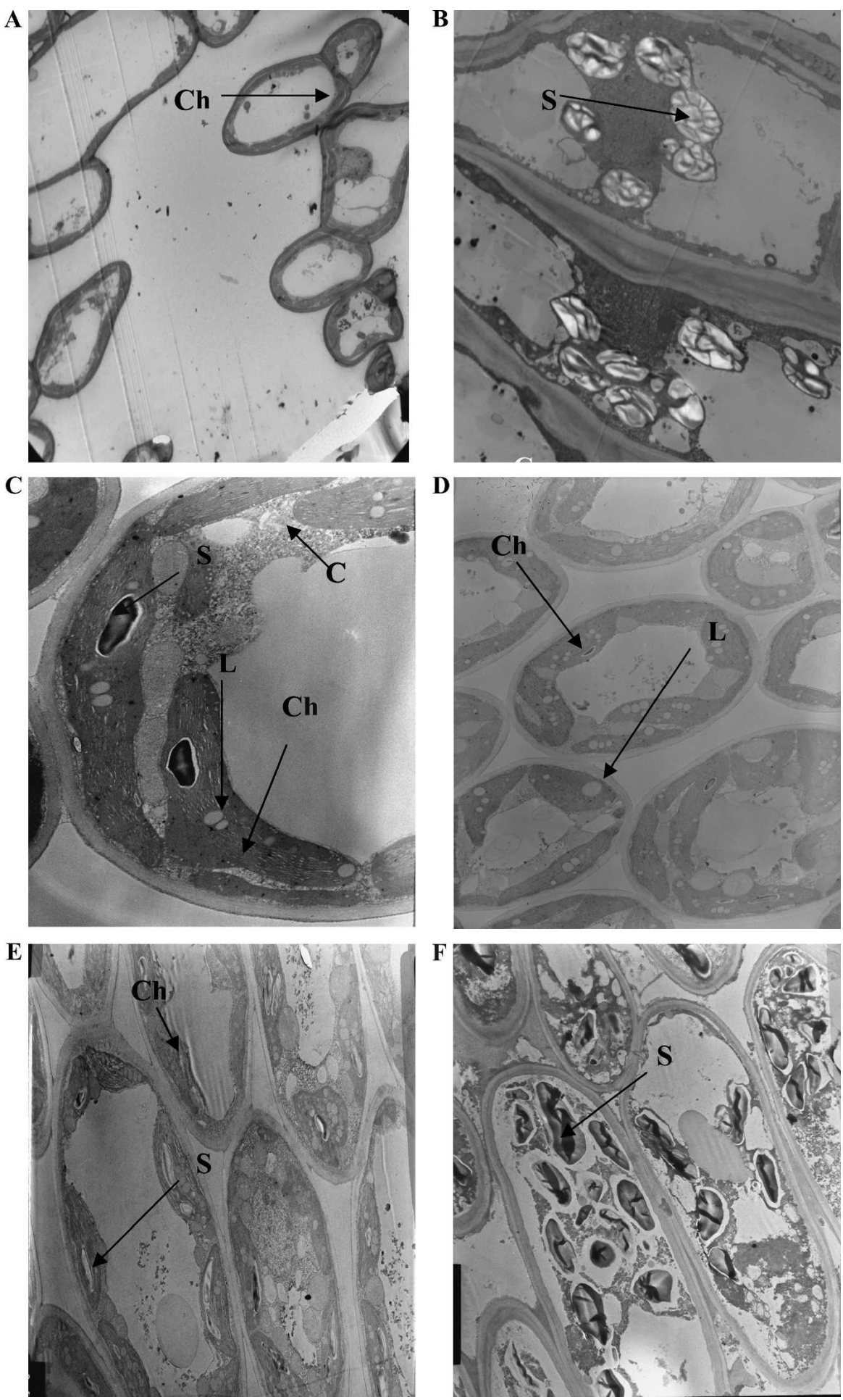

Fig. 3. Typical ultrastructure of leaves harvested in August (A, B, 2005; C-F, 2001) (A, $\times 1800)$ heavy crop load, hand thinned; $(\mathbf{B}, \times 3500)$ light crop load, hand thinned; $(\mathbf{C}, \times 4800 ; \mathbf{D}, \times 3300)$ heavy crop load, hand thinned; (E, $\times 3900)$ moderate crop load, chemical thinned; and $(\mathbf{F}, \times 3900)$ light crop load, chemical thinned. Ch, chlorosplast; C, cytoplasm; S, starch grain; L, lipid.

integrity of the chloroplast outer envelope at the earliest sampling date as seen by ruptured membranes and cytoplasm within the chloroplast (Fig. $2 \mathrm{~F}$, inset). Figure $2 \mathrm{~F}$ is from one of the trees with a chemically thinned treatment and potentially spray drift resulted in this uncharacteristic result for an early June sample.
Overall, trees with light crop loads have more discernible membrane abnormalities and accumulation of larger, more numerous starch grains before the appearance of chlorotic symptoms (Fig. 2A-E).

By August, the chloroplasts of trees with light crop loads are completely disrupted and abnormally large starch grains are found in place of chloroplasts (Figs. 3B, F). Trees with heavy and moderate crop loads showed less severe starch accumulation and less chloroplast disruption (Figs. 3A, C-E). Little discernible difference in number and size of starch grains was noted between moderate and heavy crop load. Quantification of starch will be necessary to determine if significant differences are present between these two treatments (Fig. 3D-E).

\section{Discussion and Conclusions}

Accumulation of starch grains in chloroplasts during the day is a documented function in leaves. Previously, it was thought that $\alpha$-amylase played a major role in starch cycling in chloroplasts with photosynthesis rates exceeding $\alpha$-amylase degradation of starch in chloroplasts during the day allowing a buildup of starch, which was subsequently degraded at night (Salisbury and Ross, 1991). More recently, data indicate that $\alpha$-amylase is less important for starch breakdown and other enzymes, including $\beta$-amylase and cytosolic disproportionating enzyme-like protein, are necessary to mobilize starch from the chlorosplast (Lloyd et al., 2005; Smith et al., 2004; Zeeman et al., 1998). Several factors are potentially involved in starch accumulation that leads to the ruptured chloroplasts and zonal chlorosis. Three of these potential factors are sucrose transporters, phytohormones, and enzymes. Normally, genes for photosynthesis and photosynthate mobilization are induced by carbohydrate depletion, and increases in sugar concentrations turn on genes for use and storage (Lalonde et al., 1999). A midsized gene family encodes plasma membrane sucrose transporters. Barth et al. (2003) suggest that some of these (SUT2/SUT3) may serve as flux sensors. Kuhn et al. (1997) reported SUT1 induction correlated with increased export capacity in leaves. Antisense inhibition of SUT1 resulted in carbohydrate accumulation in potato leaves, inhibition of photosynthesis, and chlorosis (Eckardt, 2003). Phytohormones are involved in increasing rate of phloem transport (Lalonde et al., 1999). A direct effect on phloem loading with indole acetic acid and gibberellic acid stimulating export of labeled assimilates was reported by Daie et al. (1986). The hormone effect appeared to be on phloem loading, not starch or sucrose formation. The role of enzymes in starch accumulation may be the result of defective amylase activity. Zeeman et al. (1998) identified a mutant with decreased endoamylase activity. Altered invertase activity was implicated in decreasing carbohydrate export and causing starch accumulation in palisade parenchyma (Das et al., 1998). In contrast, transgenic and mutant plant analyses led Lloyd et al. (2005) to conclude that plastidic glucan phosphorylase is not involved and, to a lesser extent, $\alpha$ amylase is less important in starch degradation. They report that plastidic $\beta$-amylase is important in starch mobilization and identified a new transglucosidase that inhibits starch 
breakdown. Excessive starch grain accumulation can be associated with imbalances in source/sink relationships or abnormalities with phloem loading or unloading (Chen and Cheng, 2004; Zeeman et al., 1998). Product inhibition of photosynthesis may occur as sucrose accumulates, causing a decrease in the rate of photosynthesis (Barth et al., 2003; Eckardt, 2003; Kuhn et al., 1997). However, in 'Honeycrisp', starch builds up early in the season, thus suggesting that product inhibition does not play a role in this cultivar, at least during fruit set and development.

Often the outer chloroplast membrane is among the last of the structures to be degraded in chloroplasts of senescing leaves (Biswal and Biswal, 1988). It was therefore unusual to find abnormally large, numerous starch grains and subsequent loss of integrity of the chloroplast membrane at the earliest sampling date. One would expect loss of chloroplast integrity and starch breakdown with subsequent transport of sugars to roots before leaf loss later in the season as chlorosis develops. In 'Honeycrisp', the abscission and leaf loss, although significant starch grains remain in the leaves, interrupts the normal carbon cycling and may severely limit the carbon reserves in roots over several seasons.

The occurrence of lipid bodies within chloroplasts is also associated with lateseason senescence (Smith et al., 2000). Lipid bodies are seen earlier than expected in 'Honeycrisp' trees among all treatment levels. Lipid bodies normally associated with senescent leaves in the fall are visible much sooner, in August. This may be an indication of early onset of senescence in this cultivar, unrelated to crop load.

The results presented in this study indicate that starch accumulation and loss of outer chloroplast membrane integrity occurs before symptom development. Micrographs suggest the accumulation of starch and disruption of chloroplasts is related to a decrease in crop load in 'Honeycrisp'.

Based on our findings, the zonal chlorosis of 'Honeycrisp' leaves appears to have a physiological cause related to the buildup of starch grains in the chloroplasts. This information suggests a reasonable cause of the symptoms and can be used to prevent unnecessary insecticide applications. Chlorosis, chloroplast ultrastructure, and starch accumulation are similar in trees that were thinned chemically and by hand. This suggests that chlorosis is related to crop load, not a reaction to the chemical thinners.

We propose that starch that normally would be mobilized, transporting carbon to the roots to serve as a source in the spring, is alternatively found in large grains in the disrupted chloroplasts in August. Possible hypotheses to explain why the starch accumulates abnormally in this variety of apple include: 1) excess sugar production leading to additional starch that is not cycled by the typical enzyme activity that mobilizes starch from chloroplasts; 2) potential downregula- tion or dysfunctional enzyme activity that does not mobilize starch effectively and results in detrimental levels of starch accumulation; or 3) potential downregulation or dysfunctional phloem loading/unloading preventing mobilization of sugars from the chloroplast. Results of Chen and Cheng (2004) strongly support downregulation or a dysfunctional phloem loading processes hypothesis.

Our field observations revealed unexpected variation of chlorosis symptom development during the 4 years of the study. A noticeably larger number of leaves developed chlorosis in the driest year (2005) and chlorosis was less abundant in all crop load treatments in the wettest year (2004). Growers in the mid-Hudson Valley also observed this variation (personal communication). This suggests a role for water deficit in zonal chlorosis, although water deficit also is often associated with increased sunlight and is difficult to separate in field studies. Wheeler and Fagerberg (2000) have demonstrated a role in ultraviolet $A$ and ultraviolet B levels in increasing chloroplast volume and starch granule accumulation in sunflower leaves.

Future research will be directed to include the use of a rating system to more accurately quantify chlorosis in large numbers of trees (Schupp, 2003), comparison of symptom severity with water stress in addition to crop load and quantification of starch levels throughout the diurnal period (Critchley et al., 2001). Subtractive hybridization will be implemented to compare gene expression in chlorotic and green tissue portions of leaves from trees with varying crop loads. This screening will be helpful in identification of genetic factors in 'Honeycrisp' that result in an unusual starch accumulation phenotype in trees thinned by chemical application or hand thinning. It may also provide a unique system to study carbon allocation in a tree crop.

\section{Literature Cited}

Barth, I., S. Meyer, and N. Sauer. 2003. PmSUC3: Characterization of the SUT2/SUT3-type sucrose transporter from Plantago major. Plant Cell 5:1375-1394.

Biswal, U.C. and B. Biswal. 1988. Ultrastructural modifications and biochemical changes during senescence of chloroplasts. Int. Rev. Cytol. 113:271-321

Bozzola, J.J. and L.D. Russell. 1998. Electron microscopy. 2nd Ed. Jones and Bartlett Publishers, Boston, MA.

Chen, L.S. and L. Cheng. 2004. $\mathrm{CO}_{2}$ assimilation, carbohydrate metabolism, xanthophyll cycle and the antioxidant system of 'Honeycrisp' apple leaves with zonal chlorosis. J. Amer. Soc. Hort. Sci. 129:729-737.

Critchley, J.J., S.C. Zeeman, T. Takaha, A.M. Smith, and S.M. Smith. 2001. A critical role for disproportionating enzyme in starch breakdown is revealed by knock-out mutation in Arabidopsis. Plant J. 26:89-100.

Daie, J., M. Watts, B. Aloni, and R.E. Wyse. 1986 In vitro and in vivo modification of sugar transport and translocation in celery by phytohormones. Plant Sci. 46:35-41.

Das, S.B., S.R. Bowley, and B. McKersie. 1998. A high leaf-starch mutant in alfalfa has altered invertase activity. Crop Sci. 38:722736.

Eckardt, N. 2003. The function of SUT2/SUT3 sucrose transporters: The debate continues. Plant Cell 15:1259-1262.

Kuhn, C., V.R. Franceschi, A. Schultz, R. Lemoine, and W.B. Frommer. 1997. Macromolecular trafficking indicated by localization and turnover of sucrose transporters in enucleate sieve elements. Science 275:1298-1307.

Lalonde, S., E. Boles, H. Hellman, L. Barker, J.W. Patrick, W.B. Frommer, and J.M. Ward. 1999. The dual function of plant sugar carriers in transport and in nutrient sensing. Plant Cell 11:707-726.

Lloyd, J.R., J. Kossmann, and G. Ritte. 2005. Leaf starch degradation comes out of the shadows. Trends Plant Sci. 10:130-137.

Rosenberger, D., J. Schupp, C. Watkins, K. Iungerman, S. Hoying, D. Straub, and L. Cheng. 2001. 'Honeycrisp': Promising profit maker or just another problem child? NY Fruit Quarterly 9:4-8.

Rosenberger, D.A., J.R. Schupp, S.A. Hoying, L. Cheng, and C.B. Watkins. 2004. Controlling bitter pit in 'Honeycrisp' apples. HortTechnology 14:342-349.

Salisbury, F.B. and C.W. Ross. 1991. Plant physiology. 4th Ed. Wadsworth Publishing, Belmont, CA.

Schaffer, A.A., K. Liu, E.E. Goldschmidt, C.D. Boyer, and R. Goren. 1986. Citrus leaf chlorosis induced by sink removal: Starch, nitrogen and chloroplast ultrastructure. J. Plant Phys. 124:111-121.

Schupp, J.R. 2003. Effect of chemical thinners on fruit set, yield, fruit size and fruit quality of Honeycrisp apples. NY Fruit Quart. 11:3-5.

Schupp, J.R., D.C. Ferree, and I.J. Warrington. 1992. Interactions of root pruning, and deblossoming on growth, development and yield of 'Golden Delicious' apple. J. Hort. Sci. 67:465480.

Schupp, J.R., R. Straub, D.A. Rosenberger, and C.B. Watkins. 2001. Managing 'Honeycrisp' for productivity and fruit quality. Compact Fruit Tree 34:107-109.

Smith, M.D., D.D. Licatalosi, and J.E. Thompson. 2000. Co-Association of cytochrome $f$ catabolites and plastid-lipid-associated protein with chloroplast lipid particles. Plant Phys. 124: 211-222.

Smith, S.M., D.C. Fulton, T. Chia, D. Thorneycroft, A. Chapple, H. Dunstan, C. Hylton, S.C. Zeeman, and A.M. Smith. 2004. Diurnal changes in the transcriptome encoding enzymes of starch metabolism provide evidence for both transcriptional and posttranscriptional regulation of starch metabolism in Arabidopsis leaves. Plant Phys. 136:2687-2699.

Wheeler, W.S. and W.R. Fagerberg. 2000. Exposure to low levels of photosynthetically active radiation induces rapid increases in palisade cell chloroplast volume and thylakoid surface area in sunflower (Helianthus annuus L.). Protoplasma 212:38-45.

Zeeman, S.C., F. Northrop, A.M. Smith, and T. Rees. 1998. A starch-accumulating mutant of Arabidopsis thaliana deficient in a chloroplastic starch-hydrolyzing enzyme. Plant J. $15: 357-365$ 